\section{An Unusual Presentation of Chronic Appendicitis}

An 18-year-old woman experienced intermittent uncharacterized abdominal discomfort for over a year. Cystic fibrosis had been diagnosed when she was 4 weeks old. She was maintained on substitution treatment with pancreatic enzymes and on insulin therapy because of both exocrine and endocrine pancreatic insufficiency. Involvement of the lungs required oxygen at night and systemic antibiotics four times per year because of chronic lung infection with Pseudomonas. Symptomatic cholecystolithiasis had necessitated laparoscopic cholecystectomy 4 years previously.

When she complained about discomfort in the epigastrium, gastroduodenoscopy was performed which revealed stage I gastroesophageal reflux disease. She was
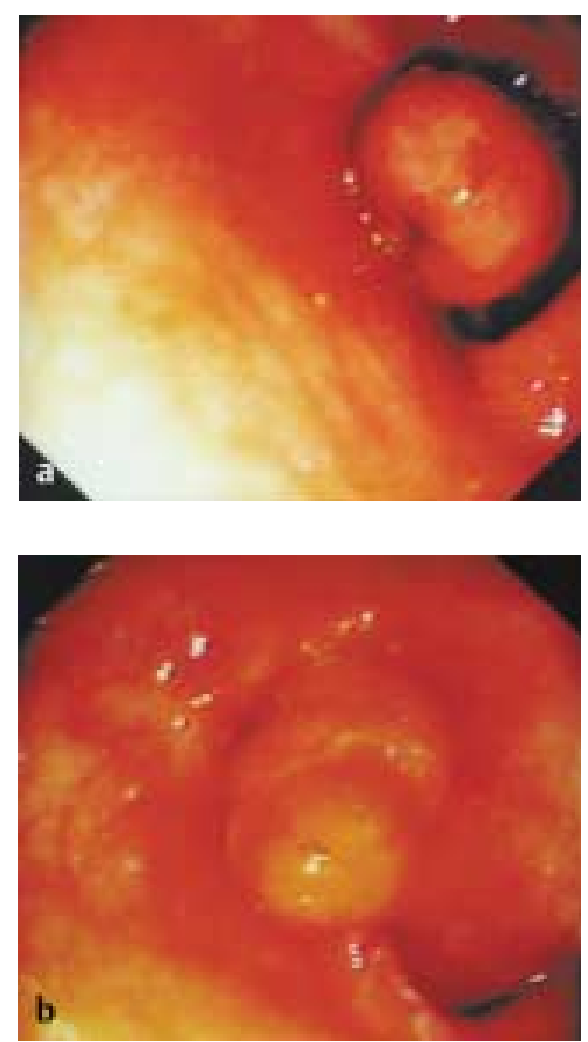

Figure 1 a,b Colonoscopy in the sigma revealed a finger-shaped structure resembling a large inverted diverticulum with adjacent signs of chronic inflammation. treated with omeprazole and her symptoms ameliorated.

At ten months later, she experienced intermittent pain in the mid-lower abdomen. Clinical and gynaecological examination as well as abdominal and vaginal ultrasound showed no pathological findings. Her symptoms improved without any specific treatment. Then 2 weeks later she complained about pain in the right upper abdomen. Sonography showed a slightly distended loop suggesting adhesions following cholecystectomy. In addition, the wall of the ascending colon appeared to be thickened, as observed in inflammatory bowel disease. All laboratory tests, except for a slightly elevated c-reactive protein level of $4.6 \mathrm{mg} / \mathrm{dl}$ (normal value $<0.5 \mathrm{mg} / \mathrm{dl}$ ), showed normal findings.

The patient underwent ileocolonoscopy, and inflammatory bowel disease as well as fibrosing colitis were excluded. However, an unusual finding was observed in the sigma (Figure $\mathbf{1}$ ).

Laparoscopy was performed and the appendix penetrating into the sigma was detected. The surgeon switched to open laparotomy and removed the appendix (Figure 2). The site of penetration into the sigma was closed without the need for resection, and the subsequent clinical course was uneventful. Pathological examination showed chronic inflammation of the appendix.

We hypothesize that the intermittent antibiotic therapy for chronic pulmonary infection had mitigated the symptoms of appendicitis, leading to this unusual course.

\section{Theilmann 1, K. Bachmann 1, P. G. Kühl² \\ ${ }^{1}$ Department of Gastroenterology, Städtisches Klinikum Pforzheim, Pforzheim, Germany \\ ${ }^{2}$ Department of Pediatrics, Städtisches Klinikum Pforzheim, Pforzheim, Germany}

\section{Corresponding Author}

\section{Theilmann, M.D.}

Medizinische Klinik II

Städtisches Klinikum Pforzheim

Kanzlerstr. 2-6

75175 Pforzheim

Germany

Fax: $\quad$ + 49-7231-969682

E-mail: Lorenz.theilmann@dgn.de

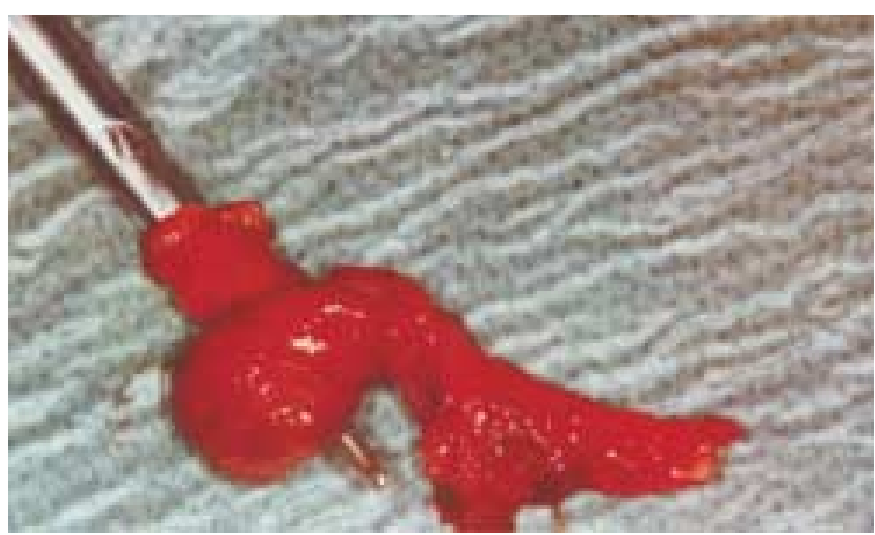

Figure 2 The resected appendix which had penetrated into the sigmoid colon. 